\title{
Value of Multislice Computerized Tomography in Evaluation of Acute Abdomen with A Hidden Cause at Sonography Mohammad T. Solyman' ${ }^{1}$ Mohammed Z. Mourad ${ }^{1}$, Nehal G. Ahmed*1, Ahmed E. Mohammed ${ }^{2}$, Sherif S. Abd Almonem ${ }^{1}$ \\ Departments of ${ }^{1}$ Radiology and ${ }^{2}$ Surgery, Faculty of Medicine, Sohag University, Sohag, Egypt. *Corresponding Author: Nehal G. Ahmed, Mobile: +201099487660, Email: nehal.ngg81@gmail.com
}

\begin{abstract}
Background: Our focus is acute abdominal pain in general, but we also discuss a number of frequently encountered urgent diagnoses in patients with acute abdominal pain: appendicitis, diverticulitis, cholecystitis, and bowel obstruction. Although perforated viscus and mesenteric ischemia are less frequently encountered, these are also addressed because imaging is of paramount importance for the timely diagnosis of these abnormalities.

Objective: Evaluation of the diagnostic accuracy of multislice Computerized Tomography (CT) examination in the diagnosis of patients presented with acute abdominal pain with a hidden cause at abdominal Ultrasonography (US) examination.

Patients and methods; the study is a prospective study which included 70 patients referred to the Radiology Department at Sohag University Hospital from the surgical emergency room with Inclusion criteria is a Patient presented with acute abdominal pain and Exclusion criteria is patient with a definite diagnosis of the cause of acute abdominal pain at the abdominal US, medical or traumatic causes of acute abdominal pain.

Results: CT imaging may be called the main method for diagnosing severe abdominal pain. When expense and ionizing radiation toxicity are key considerations, one approach is to conduct US first in all patients with severe abdominal pain, followed by CT in all instances with the non-diagnostic US. CT is much more precise and descriptive in this environment.

Conclusion: At present, CT can be considered the primary imaging technique for patients with acute abdominal pain, with the exception of patients, suspected of having acute cholecystitis. The US is preferable in these patients, but CT is an acceptable alternative.
\end{abstract}

Keywords: Multislice computerized tomography, acute abdomen, sonography.

\section{INTRODUCTION}

Acute abdomen is a surgical emergency in which there is acute and intense discomfort in the abdomen with recent onset, accompanied by abdominal-specific signs and symptoms. It refers to a broad range of diseases, from a mild and self-limiting illness to a medical emergency. Nonetheless, only around a quarter of patients historically diagnosed as having an acute abdomen undergo surgical care; therefore, the therapeutic question is determining if the patients need surgical treatment or not and, additionally, in which situations the surgical alternative should be pursued expeditiously. As a result, a rigorous and rational approach to abdominal pain diagnosis is needed ${ }^{(\mathbf{1})}$.

Rapid, reliable diagnosis is critical for reducing morbidity and mortality dramatically. Clinical evaluation is often challenging, and experimental and traditional radiologic results are frequently unspecific. Cross-sectional imaging's progress has had a significant effect on the detection and care of acute abdomen ${ }^{(2)}$. While ultrasound (US) is used in the evaluation of the biliary disease and gynecologic conditions as the initial imaging study, CT has emerged as the primary imaging modality for the evaluation of the acute abdomen ${ }^{(3)}$.

CT has been more common for evaluating abdominal pain in recent years. In 2001, nearly $10 \%$ of people presenting to emergency rooms in the United States with stomach discomfort underwent CT. By 2005 , that number increased to more than $22 \%$ of patients. Concerns over patient exposure to ionizing radiation accompany the extensive usage of $\mathrm{CT}$. In the interest of minimizing radiation exposure, abdominal CT subjects a patient to an appropriate radiation dose of about $10 \mathrm{mSv}$, relative to the annual background radiation dose of $3 \mathrm{mSv}$ in the United States; attempts have been made to utilize CT more prudently ${ }^{(4)}$.

A major disadvantage of the US is that it is an operator-dependent modality. CT was shown to be slightly more responsive than the US in identifying appendicitis and diverticulitis. For acute cholecystitis and bowel obstruction, there were no significant differences in accuracy between US and CT. The etiology of the obstruction is better evaluated with CT than with the US. Another study did not show any significant difference in accuracy between US and CT in detecting diverticulitis, but CT is more likely to detect complications of acute diverticulitis. Diverticulitis-associated abscesses are found at CT in approximately $15 \%$ of patients ${ }^{(5)}$.

The study aims to evaluate the diagnostic accuracy of multislice CT examination in diagnosing patients presented with acute abdominal pain with a hidden cause at abdominal Ultrasonography examination. 


\section{PATIENTS AND METHODS}

The study is a prospective study that included 70 patients with acute abdominal pain referred to the Radiology Department at Sohag University Hospital. From the Surgical emergency room and wards in the period between 2018 and 2019. The study was approved by the Editorial Review Board of the Radiology Department of Sohag University Hospital.

\section{Ethical considerations:}

An approval of the study was obtained from Sohag University academic and ethical committee. Every patient signed an informed written consent for acceptance of the operation.

Exclusion criteria: Patient with a definite diagnosis of the cause of acute abdominal pain at abdominal ultrasonography, medical or traumatic causes of acute abdominal pain.

All patients were subjected to:

- Abdominal and pelvic ultrasonography on a digital US scanner (Aplio 500, Toshiba Medical Systems, GE logic p9, and GE logic p5)

- Multislice CT Scanner (GE bright speed 8 slices and Toshiba Alexion 16 slices)

-The following data have been collected from all patients:

*Demographic data (Name, age, sex, residence, and occupation)

*Medical history:

- All patients presented complaining of acute abdominal pain with no age specification.

*Investigations:

- Abdominal and pelvic ultrasonography.

- Multislice CT abdominal scan examination.

- Blood laboratory tests.

\section{Statistical analysis}

Data were analyzed using SPSS version 26 (Statistical package version 26). Descriptive analysis was performed. Quantitative data were represented as mean, standard deviation, median, and range. Qualitative Data are reported as frequencies and percentages. Sensitivity, specificity, and negative and positive predictive values were determined by using contingency tables. Categorical data were analyzed using Fisher's Exact test to compare between two groups with two independent variables and Wilcoxon test to compare between two paired groups with nonparametric independent variables. Graphs were produced by using Excel or SPSS version 26. P value was considered significant if it was less than 0.05 .

\section{RESULTS}

The study was carried on 70 patients. The mean \pm SD age was $42.54 \pm 18.6$ years old. Most of the patients were females $40 / 70(57.1 \%)$ while $30 / 70$ $(42.9 \%)$ were males, with a female: male ratio $(\mathrm{F} / \mathrm{M})$ 1.3: 1. Baseline characteristics of the patients (Table 1).
Table (1): Age and gender of studied patients.

\begin{tabular}{|l|c|}
\hline Demographic data & Summary statistics \\
\hline Age/years & $42.54 \pm 18.6$ \\
Mean \pm SD & $83(2-85)$ \\
Median (range) & \\
\hline Gender & $40(57.1 \%)$ \\
Females & $30(42.9 \%)$ \\
Males & \\
\hline
\end{tabular}

(Table 2).

Results of radiological studies (US and CT)

Table (2): Results of radiological studies (US and CT) in diagnosis of acute abdominal pain proved by surgical diagnosis $(n o=70)$.

\begin{tabular}{|c|c|c|c|}
\hline \multirow{2}{*}{$\begin{array}{c}\text { Radiological } \\
\text { studies }\end{array}$} & \multicolumn{2}{|c|}{$\begin{array}{c}\text { Surgical diagnosis of } \\
\text { acute abdominal } \\
\text { pain }\end{array}$} & \multirow[t]{2}{*}{ Total } \\
\hline & $\begin{array}{c}\text { Diseased } \\
n=(62)\end{array}$ & $\begin{array}{c}\text { Free of } \\
\text { disease } \\
n=(8)\end{array}$ & \\
\hline A.US & & & \\
\hline $\begin{array}{l}\text { 1- Provisional } \\
\text { diagnosis by the US: } \\
\text { Positive } \\
\text { (Associated } \\
\text { findings) } \\
\text { Negative (No } \\
\text { associated findings) }\end{array}$ & 12 & 6 & 18 \\
\hline $\begin{array}{l}\text { 2- Definite diagnosis } \\
\text { by the US: } \\
\text { Positive (detect the } \\
\text { cause) } \\
\text { Negative (not } \\
\text { detect the cause) }\end{array}$ & $\begin{array}{l}16 \\
46\end{array}$ & $\begin{array}{l}2 \\
6\end{array}$ & $\begin{array}{l}18 \\
52\end{array}$ \\
\hline B. CT & & & \\
\hline $\begin{array}{l}\text { 1- Provisional } \\
\text { diagnosis by CT: } \\
\text { Positive } \\
\text { (Associated } \\
\text { findings) } \\
\text { Negative (No } \\
\text { associated findings) }\end{array}$ & 54 & 2 & 14 \\
\hline $\begin{array}{l}\text { 2- Definite diagnosis } \\
\text { by CT: } \\
\text { Positive (detect the } \\
\text { cause) } \\
\text { Negative (not } \\
\text { detect the cause) }\end{array}$ & $\begin{array}{c}54 \\
8\end{array}$ & $\begin{array}{l}2 \\
6\end{array}$ & $\begin{array}{l}56 \\
14\end{array}$ \\
\hline
\end{tabular}

Sensitivity, specificity, and positive and negative predictive values of ultrasonography were $25.8 \%, 71.2 \%, 88.9 \%$, and $11.5 \%$, respectively, sensitivity, specificity, and positive and negative predictive values of CT were $87.1 \%, 78.3 \%, 96.6 \%$, and $43.5 \%$, respectively (Table 3). 
Table (3): Sensitivity, specificity and accuracy of radiological manoeuvres in diagnosis of acute abdominal pain.

\begin{tabular}{|c|c|c|c|c|}
\hline $\begin{array}{r}\text { US and } \\
\text { CT } \\
\text { Parameters }\end{array}$ & $\begin{array}{c}\text { Provisional } \\
\text { diagnosis } \\
\text { of the US }\end{array}$ & $\begin{array}{c}\text { Provisional } \\
\text { diagnosis } \\
\text { of } \mathrm{CT}\end{array}$ & $\begin{array}{c}\text { Definite } \\
\text { diagnosis } \\
\text { of the US }\end{array}$ & $\begin{array}{c}\text { Definite } \\
\text { diagnosis } \\
\text { of CT }\end{array}$ \\
\hline & & & & \\
\hline & & & & $3 \%$ \\
\hline PPV & $96.2 \%$ & $96.4 \%$ & $88.9 \%$ & $96.6 \%$ \\
\hline NPV & $33.3 \%$ & $42.9 \%$ & $11.5 \%$ & $43.5 \%$ \\
\hline
\end{tabular}

\section{Illustrated cases:}

1; A 45-year-old female patient presented with acute right side abdominal pain three days post ERCP procedure. CT scan retroperitoneal retroduodenal and right paranephric air and fluid collection, Note the air is intimate to duodenal wall in A\&B images (arrows) Figure (1). Post ERCP tear of the duodenum wall is a complication of ERCP sphincterotomy for stone extraction. The duodenal content and intestinal air leak from the lumen of the duodenum into the retroperitoneal spaces, mainly retroduodenal, preaortic, and right perinephric space.
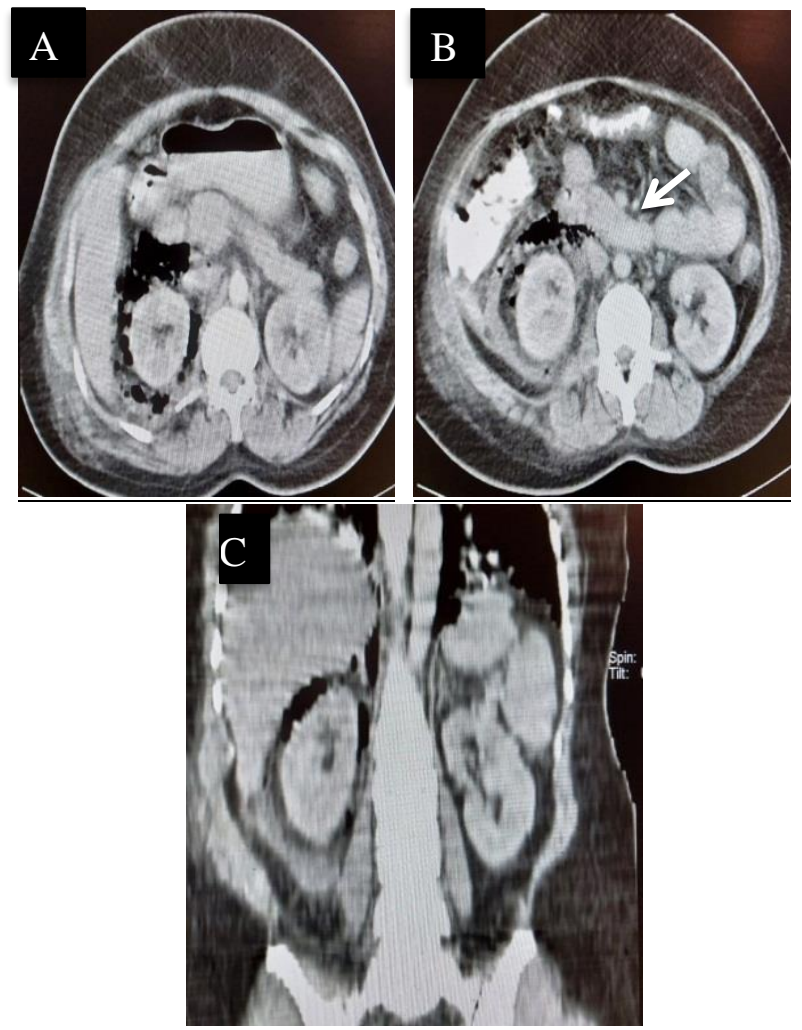

Figure (1): CT scan retroperitoneal retroduodenal and right paranephric air and fluid collection.

2; A 51-year-old male patient presented with acute diffuse abdominal pain for two days with abdominal distension and vomiting. CT scan with IV contrast; dilated small bowel loops with normal enhancing mucosa - fibrous band as a transition point causing the obstruction (red circle)- minimal free fluid collection Figure (2). The mechanical intestinal obstruction occurs when an intrinsic or extrinsic lesion closes the bowel lumen, the peristalsis keeps going trying to overcome the obstruction to some extent, and dilatation is proximal to the obstructive lesion is evident on US and CT. The lesion is a semilunar fibrous band that acts as an hook caused the small bowel obstruction.
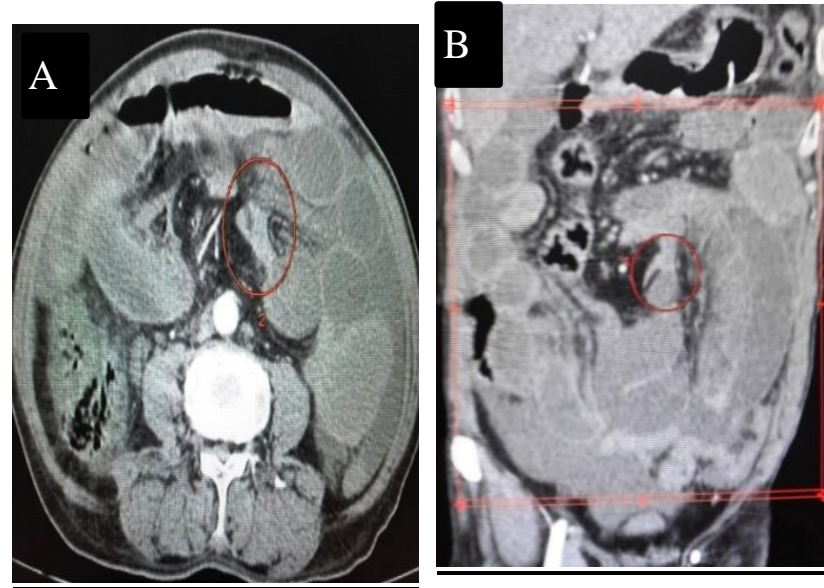

Figure (2): CT scan with IV contrast; dilated small bowel loops with normal enhancing mucosa - fibrous band.

3; A 39 year-old male patient presented with acute epigastric abdominal pain for two days referred to the back with attack of vomiting. CT scan with IV and oral contrast; CT scan revealed diffuse enlargement and homogeneous enhancement of the pancreas, with the associated minimal peripancreatic fluid collection and minimal free intraperitoneal fluid. However, no pancreatic tissue necrosis or liquefaction. The CBD and pancreatic ducts are not dilated; the splenic artery and vein are patented with normal contrast filling Figure (3).
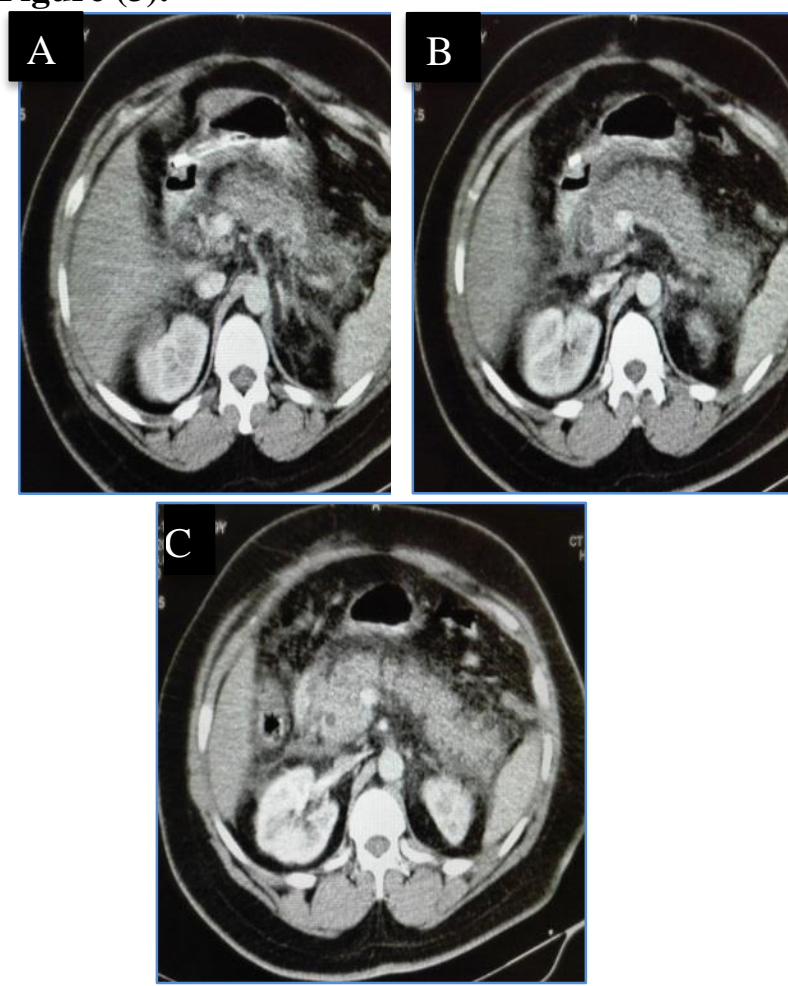

Figure (3): CT scan with IV and oral contrast revealed diffuse enlargement and homogeneous enhancement of the pancreas. 
4; A 55-year-old female patient presented with acute diffuse abdominal pain for four days with nausea and attack of vomiting. CT scan with IV and oral contrast; CT scan revealed no contrast opacification and hypodense thrombus fill the SMV and portal vein (arrows), Normal contrast filling of the SMA ( curved arrow), note the porta thrombus extended into right and left portal branches (star) Figure (4). The Mesenteric vascular occlusion resulted in bowel congestion, ischemia, and non-mechanical obstruction. It manifests when mesenteric arterial occlusion or mesenteric venous occlusion occurs. The earlier the diagnosis, the better results after treatment and the better response to medical thrombolytic therapy.
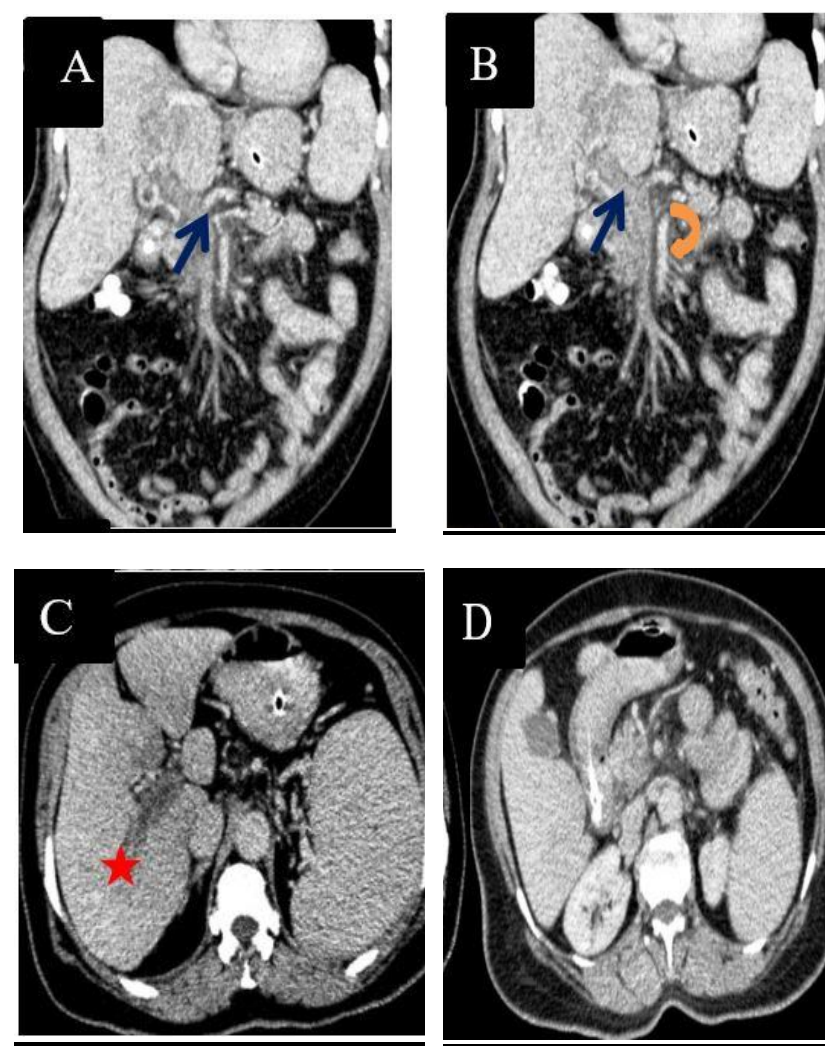

Figure (4): CT scan with IV and oral contrast revealed no contrast opacification and hypodense thrombus fill the SMV and porta vein.

5; A 72-year-old male patient presented with acute diffuse abdominal pain for one day with nausea and attack of vomiting. CT scan with IV and oral contrast; CT scan revealed whirlpool sign of the mesenteric vessels (arrows) with compressed attenuated mesenteric vein but normal mesenteric artery (A), that was associated with lower abdomen small bowel (ileal) mild wall edema (D) Figure (5).

The Mesenteric volvulus is an abnormal rotation of the mesentery or part of it around its axis resulted in venous obstruction early and arterial occlusion later if it was neglected. The effect on the bowel is ischemia, either venous or arterial, with signs of functional intestinal obstruction; neglected cases would show signs of peritonitis.
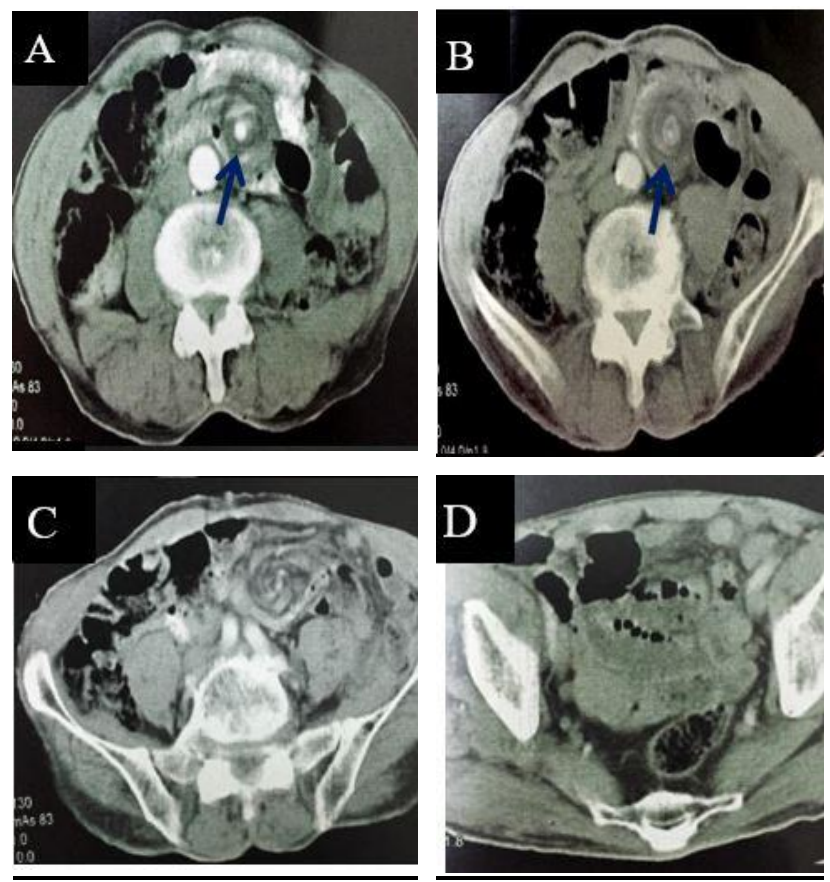

Figure (5): CT scan with IV and oral contrast revealed whirl pool sign of the mesenteric vessels (arrows) with compressed attenuated mesenteric vein but normal mesenteric artery (A), that was associated with lower abdomen small bowel (ileal) mild wall edema (D).

\section{DISCUSSION}

The aim of our study is the evaluation of the diagnostic accuracy of multislice CT examination in the diagnosis of patients presented with acute abdominal pain with a hidden cause at abdominal ultrasonography examination.

The study was carried on 70 patients with a mean age of $42.54 \pm 18.6$ years old. Most of the patients were females $40 / 70(57.1 \%)$ while $30 / 70$ (42.9\%) were males, with a female: male ratio $(\mathrm{F} / \mathrm{M}) 1.3: 1$.

We evaluated the various causes of acute abdomen. Common diagnoses were acute intestinal obstruction due to various causes in $28(40 \%)$, acute appendicitis in $10(14.3 \%)$, and inflammation of the bowel as IBD and diverticulitis in10 (14.3\%). Other causes were ureteric stones, pancreatitis, and ruptured ovarian cysts.

In our study, the sensitivity, specificity, and positive and negative predictive values of MDCT were $87.1 \%, 78.3 \%, 96.6 \%$, and $43.5 \%$, respectively, which was comparable to the study results of Rafiq et al. ${ }^{(6)}$ in which the sensitivity, specificity and positive and negative predictive values of MDCT were $95.0 \%$, $75 \%, 98.3 \%$, and $60 \%$ respectively. In our study, sensitivity, specificity, and positive and negative predictive values of ultrasonography were $25.8 \%$, $71.2 \%, 88.9 \%$, and $11.5 \%$, respectively.

The effectiveness of US in diagnosing the precise cause of bowel obstruction is marginal, ranging between $28 \%$ and $46 \%$ (7 and 8 ). As with previous research, our findings indicate that the US is of minimal use for detecting bowel strictures with various 
etiologies or obstructions caused by adhesions or malrotation.

Some cases of intussusception could be missed by the US due to obesity or gaseous distension, while CT can easily diagnose it. Best known is the so-called bowel-within-bowel configuration, in which the layers of the bowel are duplicated, forming concentric rings (target sign) when imaged at right angles to the lumen, and a soft tissue sausage when imaged longitudinally ${ }^{(7)}$.

CT is exceptionally receptive (94-98 percent sensitivity) and precise (up to 97 percent specificity) for the diagnosis of acute appendicitis and also allows for the diagnosis of possible causes of abdominal pain. Contrast (IV, oral, or both) is debatable and differs by organization. Oral comparison has not been shown to improve CT sensitivity ${ }^{(9)}$. Moreover, this is consistent with our research, which found that CT has a sensitivity of 96.6 percent and a precision of 99.1 percent for diagnosing acute appendicitis.

The US is believed to have a significant drawback due to its observer-dependent nature. Its precision, as recorded in the literature, can be overstated, as the US is typically conducted by highly trained observers in a testing setting. Additionally, US accuracy may be reduced in some patient subgroups, such as obese patients, males, and certain age groups, especially women of reproductive age. CT, on the other side, has an excellent inter-observer arrangement with repeated diagnoses causing severe abdominal distress (e.g., appendicitis and diverticulitis) ${ }^{(10)}$.

In most cases of our study, US limitations were due to gaseous distension associated with bowel obstruction and obesity, especially in appendicitis cases.

CT is sensitive for the diagnosis of acute female genital conditions. MRI is superior to CT for further characterization of acute disorders such as adnexal torsion, corpus luteum, and hemorrhagic cysts ${ }^{(\mathbf{1 0})}$.

Although historically, catheter angiography was the gold standard for imaging of suspected intestinal ischemia, CT has replaced it with its ability to volumetrically assess the whole abdomen in multiple vascular phases, e.g., arterial, portal venous, and delayed. Ischemic segments have been found to have lower densities and iodine concentrations compared to non-ischemic segments ${ }^{(11)}$.

\section{CONCLUSION}

MDCT has higher sensitivity, specificity, and accuracy rate in the diagnosis of acute abdomen than US. In inconclusive cases, MDCT is recommended to arrive at a definitive diagnosis. The results obtained in the study were comparable to pioneer studies conducted worldwide.

\section{REFERENCES}

1. Mazzei M, Guerrini S, Squitieri N et al. (2013): The role of US examination in the management of acute abdomen. Critical Ultrasound Journal, 5:1-9.

2. El Kheshen A (2012): Role of MSCT (multislice computed tomography) in acute abdomen using 64-slice technology. The Egyptian Journal of Radiology and Nuclear Medicine, 43:41-51.

3. Cartwright S, Knudson M (2015): Diagnostic imaging of acute abdominal pain in adults. American Family Physician, 91:452-9.

4. Kammerer S, Schuelke C, Berkemeyer S et al. (2018): The role of multislice computed tomography (MSCT) angiography in the diagnosis and therapy of non-occlusive mesenteric ischemia (NOMI): Could MSCT replace DSA in diagnosis? PLoS One, 13:193698.

5. Rafiq S, Ellahi I, Shabir S et al. (2019): Multidetector computerized tomography in acute abdomen. International Surgery Journal, 6:2353-2357.

6. Sheedy S, Earnest F, Fletcher J et al. (2006): CT of small-bowel ischemia associated with obstruction in emergency department patients: diagnostic performance evaluation. Radiology, 241:729-36.

7. Meiser G, Meissner K (1987): Ileus and intestinal obstruction--ultrasonographic findings as a guideline to therapy. Hepato-gastroenterology, 34:194-9.

8. Anderson S, Soto J, Lucey B et al. (2009): Abdominal 64-MDCT for suspected appendicitis: the use of oral and IV contrast material versus IV contrast material only. American Journal of Roentgenology, 193:1282-8.

9. Roche O, Chavan N, Aquilina J et al. (2012): Radiological appearances of gynaecological emergencies. Insights Into Imaging, 3:265-75.

10.Lourenco P, Rawski R, Mohammed M et al. (2018): Dual-energy CT iodine mapping and 40-keV monoenergetic applications in the diagnosis of acute bowel ischemia. American Journal of Roentgenology, 211:564-70.

11.Lourenco P, Rawski R, Mohammed M et al. (2018): Dual-Energy CT Iodine Mapping and 40-keV Monoenergetic Applications in the Diagnosis of Acute Bowel Ischemia. AJR Am J Roentgenol., 211:564-70. 